\title{
Enfermedad de Kawasaki: una serie clínica
}

\author{
Isolda Budnik O., Tamara Hirsch B., Carlos Fernández C., Leticia Yánez P. y Juanita Zamorano R.
}

\footnotetext{
Universidad de los Andes, Santiago, Chile. Facultad de Medicina (IBO, JZR). Clínica Santa María, Santiago, Chile. nfectología Pediátrica (THB, JZR). Unidad de Pacientes Críticos Pediátrica (CFC, LYP) Servicio de Urgencia (CFC)

Establecimiento donde se realizó el trabajo: Clínica Santa María

Los autores declaran no tener conflictos de interés

Fuente de financiamiento: no hubo

Recibido: 8 de diciembre de 2010 Aceptado: 17 de mayo de 2011

Correspondencia a: Isolda Budnik Ojeda isolbudnik@gmail.com
}

\section{Kawasaki disease: a clinical serie}

Introduction: Kawasaki disease (KD) is a serious disease in children due to its potential complications and sequelae if not promptly and adequately managed. Objectives: To describe clinical and epidemiological characteristics of children hospitalized due to KD at a tertiary care center and identify risk factors for poor outcome. Patients and Methods: Retrospective and descriptive study of 32 medical records of patients hospitalized with diagnosis of KD at a tertiary care center of Santiago, Chile between February 1999 and May 2007. Results: The annual frequency was of 5 cases, mainly boys and during spring. The median age at diagnosis was 1.5 years and $87.5 \%$ of the children were younger than 5 years. Typical presentation prevailed in all ages $(68,7 \%)$. Coronary artery affection, including dilatation or aneurisms, occurred in $21.9 \%$ of the cases, with aneurysms in 3 cases. All patients were treated with aspirin and intravenous immunoglobulin (IVIG); 4 patients required a second dose. No deaths were reported. The identified risk factors for poor outcome were age older than 5 years and hypoalbuminemia. Conclusions: KD is an infrequent disease that mainly occurs in children younger than 5 years and with a typical presentation. There are risk factors associated with poor outcome.

Key words: Kawasaki disease, coronary artery, immunoglobulin.

Palabras clave: Enfermedad de Kawasaki, arteria coronaria, inmunoglobulina.

\section{Introducción}

L a enfermedad de Kawasaki (EK) es una vasculitis sistémica de la infancia, que afecta predominantemente a niños bajo cuatro años de edad y cuya etiología aún es desconocida. Dadas sus características clínicas y epidemiológicas, se ha planteado la asociación con agentes infecciosos; debido a las diversas incidencias observadas en distintos grupos étnicos, con claro predominio en el continente asiático ${ }^{1}$, también se postula la existencia de una predisposición genética.

Esta patología se presenta en todo el mundo y se considera que es la causa más frecuente de isquemia miocárdica en niños ${ }^{2}$. La incidencia de la EK, varía entre países, estimándose en 90/100.000 niños bajo cinco años de edad en Japón, 8,9/100.000 en Estados Unidos y 3,7/100.000 en Australia ${ }^{3-5}$. En Chile, las publicaciones sobre este tema son escasas, registrándose reportes de incidencia entre 3 y 3,9/100.000 6 .

La frecuencia descrita internacionalmente de aneurismas coronarios o de desarrollo de ectasia en pacientes no tratados con inmunoglobulina humana intravenosa (IGIV), es de 15 a 25\%; el tratamiento con IGIV en altas dosis ha demostrado eficacia en reducir la aparición de anomalías de arterias coronarias hasta 2 a $4 \%^{2-8}$. La mortalidad descrita en pacientes con EK tratados con IGIV es de $0,04 \%$.

El objetivo de este trabajo es describir la experiencia de nuestro Servicio de Pediatría en el diagnóstico y tra- tamiento de enfermedad de Kawasaki durante ocho años e identificar factores de riesgo presentes al ingreso en los casos de evolución más grave.

\section{Pacientes y Métodos}

Revisión de 32 fichas de pacientes con diagnóstico de egreso de EK (febrero de 1999-mayo de 2007) en la Clínica Santa María. La información se recolectó en un formulario ad-hoc. Se consideraron datos bio-demográficos y las siguientes características clínicas: días de evolución del cuadro previo a la consulta, días de duración de la fiebre, días de hospitalización, diagnóstico de ingreso y de egreso, tiempo de demora en el diagnóstico de EK desde la consulta en el Servicio de Urgencia, co-morbilidad, exámenes de laboratorio, terapias utilizadas, evolución clínica y el resultado final (complicaciones, necesidad de repetir dosis de inmunoglobulina y secuelas cardíacas o extra-cardíacas). La revisión se realizó con la aprobación del Comité de Ética de la institución.

\section{Definición de casos}

Los casos se clasificaron según su forma de presentación, en típico, atípico e incompleto de acuerdo a los criterios del Committee of Rheumatic Fever, Endocarditis, and Kawasaki Disease de la American Heart Association $^{2}$. Se consideró típico aquel que presentaba al menos cuatro de los cinco ítems del criterio clásico ${ }^{2}$; 
incompleto, aquel que tenía menos de cuatro y atípico, aquel con manifestaciones infrecuentes ${ }^{2}$.

\section{Definición de parámetros de laboratorio y de EK graves}

Se consideró anemia: valores de hemoglobina bajo 2 DE para edad y sexo; leucocitosis: recuentos de leucocitos $>15.000 / \mathrm{mm}^{3}$; trombocitosis: recuentos de plaquetas $>400.000 / \mathrm{mm}^{3}$; velocidad de eritrosedimentación (VHS) elevada: $>30 \mathrm{~mm} / \mathrm{h}$ y proteína $\mathrm{C}$ reactiva (PCR) elevada: valores $>50 \mathrm{mg} / \mathrm{L}$.

Todos los pacientes con criterios de sospecha de EK fueron sometidos a ecocardiograma Doppler color efectuado por un cardiólogo entrenado y con ecocardiógrafos HP SONOS 800 y GE Vivid Seven. Este examen se repitió de acuerdo a protocolos de seguimiento previamente publicados $^{3}$.

La definición de compromiso coronario se efectuó según criterios ecocardiográficos descritos en las guías de práctica clínicas publicadas por la American Heart Association $2006^{3}$.

Se consideró enfermedad de Kawasaki grave a aquellos casos con dilatación o aneurismas coronarios, aquellos que requirieron una segunda dosis de inmunoglobulina y/o tiempo de hospitalización mayor de 7 días.

\section{Análisis estadístico}

El análisis de la información se hizo por el test de pruebas pareadas y test de $t$ de student para variables independientes. Para el análisis de los factores de riesgo se utilizó Odds Ratio y se consideró un valor de $\mathrm{p}$ significativo $<0,05$.

\section{Resultados}

\section{Características bio-demográficas}

Para los 32 pacientes estudiados se pueden observar en la Tabla 1. La frecuencia anual de casos varió entre 3 y 8 al año. Diecinueve niños (59\%) fueron hombres, con una relación hombre: mujer $=1,46: 1$. El rango de edad fluctuó entre 5 meses y 8 años 9 meses. Tenían bajo 5 años de edad $87,5 \%$ y bajo 2 años $68 \%$.

Los casos de EK se concentraron en primavera

Tabla 1. Características bio-demográficas en 32 casos de enfermedad de Kawasaki

\begin{tabular}{|lcc|}
\hline Característica & $n$ de casos & $\%$ \\
\hline Femenino & 13 & 41 \\
Masculino & 19 & 59 \\
$<2$ año & 22 & 68,8 \\
1 a 5 años & 6 & 18,7 \\
$>5$ años & 4 & 12,5 \\
\hline
\end{tabular}

Tabla 2. Frecuencia de síntomas y signos clásicos presentes en 32 casos de enfermedad de Kawasaki

\begin{tabular}{|lcc|}
\hline Criterios & $n$ de casos & $\%$ \\
\hline Fiebre $>5$ días & 28 & 87,5 \\
\hline Exantema & 28 & 87,5 \\
\hline Compromiso de la mucosa oral & 28 & 87,5 \\
\hline Inyección conjuntival & 28 & 87,5 \\
\hline Compromiso de extremidades & 26 & 81,3 \\
\hline Adenopatía cervical & 18 & 56,2 \\
\hline
\end{tabular}

$(51,6 \%)$, seguido por el otoño (29\%), verano (12,9\%) e invierno $(6,4 \%)$.

\section{Características clínicas}

El 75\% de los casos se presentó como EK típica, 21,8\% como incompleta y 3,2\% como atípica. El diagnóstico se realizó en promedio a los 5,21 días de enfermedad, con rango entre 1 y 15 días. Al momento de la hospitalización, $46,8 \%$ de los pacientes presentó fiebre por menos de cinco días (en la mitad de éstos casos se planteó el diagnóstico antes del quinto día de fiebre) y todos tuvieron fiebre por cinco días o más durante la evolución de la enfermedad. La frecuencia de los signos y síntomas que definen el criterio diagnóstico se describe en la Tabla 2. Los más frecuentes fueron fiebre, conjuntivitis, exantema y compromiso de mucosa oral y el menos frecuente la adenopatía cervical. Entre las alteraciones de extremidades, se presentó exantema en 18 pacientes $(56,2 \%)$, edema en 19 $(59,3 \%)$ y descamación peri-ungueal en 11 (34,3\%). En 15 casos el exantema fue maculo-papular, en siete macular, en cuatro escarlatiniforme y en dos polimorfo (purpúrico y maculo-papular). La descamación fue observada en seis casos durante la primera semana de hospitalización, en cuatro casos durante la segunda semana y en un caso durante la tercera semana de hospitalización.

Al relacionar las formas de presentación con los grupos etáreos, se observó que bajo 2 años de edad, 72,7\% presentó una EK típica, 27,3\% una EK incompleta y no hubo casos atípicos. En el grupo entre 2 y 5 años, los porcentajes fueron de $66,6 \%, 16,7 \%$ y $16,7 \%$, respectivamente. Sobre los 5 años de edad, la EK se presentó en forma típica en todos los pacientes.

El 90,6\% de los niños presentó al menos uno de los siguientes signos/síntomas: artritis o artralgias, irritabilidad, compromiso gastrointestinal, hepatitis y hepatomegalia, hidrops vesicular, meningitis aséptica, eritema e induración del sitio de la vacuna BCG y, con menor frecuencia, edema escrotal, nódulos e infiltrados pulmonares, efusión pleural y síndrome hemofagocítico, observándose irritabilidad en 37,5\% de los casos y eritema en la cicatriz de la vacuna BCG en 34,3\%. 


\section{Experiencia Clínica}

Tabla 3. Complicaciones y co-morbilidad en 32 casos de enfermedad de Kawasaki

\begin{tabular}{|lcc|}
\hline Complicación & $n$ de casos & $\%$ \\
\hline Shock mixto & 4 & 12,5 \\
\hline Shock cardiogénico & 2 & 6,2 \\
\hline Síndrome de activación macrofágica & 1 & 3,1 \\
\hline Meningitis aséptica & 1 & 3,1 \\
\hline Granuloma hepático & 1 & 3,1 \\
\hline Adenoflegmón cervical bacteriano & 1 & 3,1 \\
\hline Vasculitis de piel & 1 & 3,1 \\
\hline Pielonefritis aguda por Escherichia coli & 1 & 3,1 \\
\hline Co-infección por virus respiratorio sincicial & 1 & 3,1 \\
\hline Co-infección por parvovirus B19 y enterovirus & 1 & 3,1 \\
\hline
\end{tabular}

\section{Exámenes de laboratorio}

Al ingreso, presentaron anemia 15 niños $(48,3 \%)$ y trombocitopenia un solo caso. En 13 niños $(40,6 \%)$ se encontró leucocitosis. Treinta (93,5\%) registraron una VHS al ingreso mayor a $30 \mathrm{~mm} / \mathrm{h}$, con una mediana de $76 \mathrm{~mm} / \mathrm{h}$ (13-134). La PCR fue mayor a $50 \mathrm{mg} / \mathrm{L}$ en 21 (66\%), con una mediana de $103 \mathrm{mg} / \mathrm{L}$ (54-220).

Otras alteraciones de los parámetros de laboratorio fueron elevación de transaminasas (SGOT) $(34,4 \%)$, hipoalbuminemia $(25 \%)$, alteraciones de pruebas de coagulación $(21,8 \%)$, piuria estéril $(9,3 \%)$ y creatininemia mayor a $1,5 \mathrm{mg} / \mathrm{dl}(6,2 \%)$.

\section{Ecocardiograma Doppler color}

El total de casos que presentó algún tipo de alteración ecocardiográfica descrita en la EK fue $17(53,1 \%)$. Se realizó ecocardiograma de ingreso a todos los niños, encontrando alteraciones precoces en 12 de ellos. Entre ellas, hubo seis casos con efusión pericárdica (dos como único hallazgo, dos asociados a refringencia coronaria, uno a dilatación coronaria y uno con disminución de la contractibilidad del ventrículo izquierdo), dos casos de eco-refringencia aislada, dos casos con eco-refringencia asociada a dilatación coronaria, un caso con dilatación coronaria como única anormalidad y otro con disminución de la contractibilidad del ventrículo izquierdo. Durante la primera semana, en un segundo ecocardiograma, tres casos presentaron alteraciones eco-cardiográficas nuevas, todos con efusión pericárdica (único hallazgo, asociado a insuficiencia valvular mitral y a disfunción ventricular izquierda, respectivamente). El paciente que presentó dilatación coronaria como única lesión en el ecocardiograma de ingreso, evolucionó hacia un aneurisma gigante. Durante la segunda semana, en dos casos apareció dilatación coronaria (uno de ellos asociada a efusión pericárdica). Por último, dos de los niños que habían presentado efusión pericárdica y dilatación coronaria durante la hospitaliza- ción, evolucionaron hacia la formación de aneurismas, llegando a un total de $9,4 \%$.

\section{Tratamiento}

Durante la hospitalización, todos los pacientes recibieron ácido acetilsalicílico ( 80 a $100 \mathrm{mg} / \mathrm{kg} /$ día) e IGIV $2 \mathrm{gr} / \mathrm{kg}$-peso. En cuatro casos $(12,5 \%)$ se requirió una segunda dosis de esta última, siendo tres de ellos sobre 5 años de edad. Hubo once casos en los que se utilizó antimicrobianos al inicio del cuadro, por sospecha de infección bacteriana previo al diagnóstico de EK. Tres niños recibieron corticosteroides iv como terapia para shock refractario.

\section{Complicaciones}

Seis pacientes evolucionaron con shock, que se presentó en dos casos al ingreso y en cuatro casos durante la evolución (Tabla 3). De ellos, dos presentaron shock cardiogénico y cuatro mixtos. Otras complicaciones o comorbilidades observadas fueron síndrome de activación macrofágica, meningitis aséptica, granuloma hepático, adenoflegmón cervical bacteriano (no se aisló agente etiológico), vasculitis de piel, pielonefritis aguda por Escherichia coli, infección por virus respiratorio sincicial y co-infección por parvovirus B19 y enterovirus (no tipificado), en un caso cada uno.

\section{Pronóstico y factores de riesgo}

El seguimiento de los pacientes ha sido variable en tiempo y adherencia, prácticamente sin aparición de nuevas alteraciones en pacientes que al egreso no tenían aneurismas. De los tres pacientes con aneurismas al momento del alta, uno evolucionó con desaparición de éstos al cabo de cuatro años y en los otros dos hubo persistencia de los aneurismas hasta junio de 2010 y enero del 2011, respectivamente. No hubo letalidad en esta serie al momento de enviar el manuscrito a publicación.

Hubo siete casos de EK grave, por presencia de aneurismas, hospitalización mayor a 7 días o más de una dosis de IGIV, en dos casos con más de uno de ellos. Se identificó como factores de riesgo de enfermedad grave la edad mayor a 5 años (OR: $18(1,13-607)$ ) y la presencia de hipoalbuminemia menor a 3,5 g/dl (OR: 9,78 (1,06$112,9)$ ), ambos con valores de $\mathrm{p}<0,05$ (Tabla 4).

\section{Discusión}

Los hallazgos en esta serie de pacientes con EK coinciden con lo descrito en la literatura médica internacional.

La EK es una vasculitis sistémica, febril, de etiología desconocida, con una incidencia que varía según la localización geográfica. En nuestra serie, la frecuencia anual varió entre 3 y 8 casos (promedio de 4), aproximadamente $0,1 \%$ del total de egresos pediátricos anuales en 
Tabla 4. Factores de riesgo para mala evolución en 32 casos de enfermedad de Kawasaki

\begin{tabular}{|lcccc|}
\hline Factores de riesgo & EK grave* $(n=7)^{\text {EK leve* }(n=25)}$ & Odds Ratio & $p$ value \\
\hline Edad $>5$ años & 3 & 1 & $18(1,13-607)$ & 0,02 \\
Sexo masculino & 4 & 15 & $0,89(0,12-6,55)$ & 0,6 \\
\hline Presentación típica & 6 & 16 & $3,39(0,3-86,76)$ & 0,2 \\
Recuento plaquetas de ingreso $>400.000 / \mathrm{mm}^{3}$ & 2 & 1 & $9,6(0,58-338,1)$ & 0,11 \\
\hline Recuento de leucocitos al ingreso $>15.000 / \mathrm{mm}^{3}$ & 2 & 10 & $0,6(0,06-4,74)$ & 0,4 \\
VHS $>100 \mathrm{~mm} / \mathrm{h}$ & 4 & 5 & $5,33(0,67-47,51)$ & 0,07 \\
\hline Hipoalbuminemia & 4 & 3 & $9,78(1,06-112,9)$ & 0,02 \\
Presencia de BCGitis & 2 & 9 & $0,71(0,08-5,7)$ & 0,5 \\
\hline *Definición en Material y Métodos. & & & & \\
\hline
\end{tabular}

la institución, lo que se asemeja a los datos publicados por Gentles y cols, en Nueva Zelandia ${ }^{10}$. La distribución según género y edad fue similar a la descrita en trabajos australianos, japoneses, coreanos, neozelandeses, americanos y chilenos, describiéndose predominantemente en prescolares de género masculino ${ }^{2,4,5,7,9-11} ; 87,5 \%$ de los casos de nuestra serie tenían menos de 5 años, con razón de predominio masculino de 1,46:1. En lo que respecta a la aparición estacional de la EK, se observó una diferencia parcial con los datos aportados en los diferentes estudios. Las estaciones más comúnmente descritas son primavera e invierno ${ }^{2}$. En nuestra serie hubo claro predominio en primavera.

El diagnóstico de la EK es esencialmente clínico y no se debe retrasar el diagnóstico ni el tratamiento a la espera de la aparición de todos los signos y síntomas. Nosotros observamos predominio de la presentación típica de la EK en todas las edades, siendo la forma incompleta segunda en frecuencia bajo los 2 años de edad. Estos datos coinciden con los de otros reportes en la literatura médica $^{5-12}$ que describen un aumento de EK incompleta en el concierto mundial; en nuestra serie fue $18,7 \%$, semejante a lo descrito ${ }^{4,5}$.

La fiebre en la EK es típicamente alta $\left(39\right.$ a $\left.40^{\circ} \mathrm{C}\right)$ y mayor o igual a cinco días. En nuestra serie la mediana de días con fiebre fue de cinco días, con un rango entre uno y 15 días. Cincuenta y tres por ciento de los casos presentó fiebre por al menos cinco días, previo al diagnóstico.

Las alteraciones en las extremidades son características muy orientadoras en el diagnóstico de EK, sobre todo en conjunto con el resto de la sintomatología. El eritema de manos y pies o edema, algunas veces doloroso, ocurre generalmente en la fase más aguda de la enfermedad. La descamación, de inicio peri-ungueal, en los dedos de la manos o de los pies, usualmente es más tardía, apareciendo frecuentemente en la tercera semana desde el inicio de la fiebre. Otras alteraciones, tales como surcos en las uñas, pueden aparecer de manera aún más tardía, aproximadamente 1 a 2 meses post inicio de la fiebre. En nuestra serie se observó que 81,2\% de los pacientes tenían manifestaciones en las extremidades, desarrolladas durante el transcurso de la enfermedad; creemos que si se realizara un seguimiento de los pacientes, posterior al alta, este porcentaje sería aún mayor.

El compromiso cutáneo típico consiste en un rash escarlatiniforme, no vesicular, que aparece precozmente, en los cinco primeros días luego de iniciada la fiebre. Se presenta inicialmente en el tronco y luego se disemina comprometiendo extremidades, acentuándose en la región perineal, donde a veces se produce descamación. En nuestro estudio $87,5 \%$ de los niños presentó algún tipo de exantema.

La inyección conjuntival bilateral, que compromete con mayor frecuencia a la conjuntiva bulbar, también es de inicio precoz. No se asocia a exudado, edema conjuntival o a úlceras corneales y es generalmente indolora. Se puede asociar a iridociclitis aguda moderada o a uveítis anterior, generalmente ambas son de rápida resolución. En nuestra serie, $87,5 \%$ de los pacientes presentó inyección conjuntival, sin otras complicaciones.

En los labios y la mucosa orofaríngea se producen cambios tempranamente, incluyendo labios rojos, secos, fisurados y descamados, que incluso pueden sangrar. La lengua se observa eritematosa, con papilas prominentes y eritema difuso que le da un aspecto "aframbuesado". En nuestro estudio, este tipo de alteraciones se registró en $87,5 \%$ de los casos.

La linfoadenopatía cervical es el signo que se presenta con menor frecuencia en todas las series ${ }^{3}$. Generalmente es unilateral, se localiza en el triángulo cervical anterior y es mayor a $1,5 \mathrm{~cm}$ de diámetro. En nuestra serie este signo estuvo presente en $56,2 \%$ de los pacientes, similar a lo descrito por otros autores.

Otros síntomas y signos que se observan en la EK son: artritis o artralgias de pequeñas y grandes articulaciones, simétricas y transitorias, preferentemente en niños mayo- 
res y durante la primera semana de evolución; irritabilidad importante, especialmente en lactantes, compromiso gastrointestinal variable (diarrea, vómitos y dolor abdominal en aproximadamente $1 / 3$ de los pacientes), hepatitis y hepatomegalia, hidrops vesicular (aproximadamente en 1/6 de los pacientes en las primeras dos semanas), meningitis aséptica (en aproximadamente la mitad de los casos en que se realiza punción lumbar), eritema e induración en torno a la cicatriz de la vacuna BCG. Menos frecuentemente se observa edema escrotal, nódulos e infiltrados pulmonares, efusión pleural y síndrome hemofagocítico. En nuestra serie, 90,6\% de los niños presentó al menos uno de estos signos clínicos, observándose con mayor frecuencia la irritabilidad y eritema en la cicatriz de la vacuna BCG.

El uso de exámenes de laboratorio como condicionantes para el diagnóstico de la EK, es un error conceptual. $\mathrm{Si}$ bien existen ciertos parámetros característicos que se dan en el contexto de esta patología, éstos son inespecíficos y pueden no estar presentes. Cabe destacar que es importante buscarlos y controlarlos, tanto al ingreso como durante la evolución del paciente para detectar precozmente las complicaciones. Alteraciones de pruebas de laboratorio frecuentes y sugerentes pero no patognomónicas de EK se aprecian en el hemograma, las pruebas de función hepática, el perfil bioquímico y en el examen de sedimento urinario. El hemograma suele mostrar leucocitosis con predominio de formas inmaduras en la etapa aguda -se presenta en aproximadamente la mitad de los pacientes- $\mathrm{y}$ anemia, trombocitosis en la segunda semana de evolución, probablemente el cambio más constante, y rara vez trombocitopenia. La aparición precoz de esta última se ha descrito como un factor de riesgo para el desarrollo de aneurismas coronarios y mala evolución ${ }^{2}$. En nuestro análisis hubo leucocitosis en sólo $1 / 3$ de los casos, anemia en $80,7 \%$, trombocitosis en $37 \%$ y trombocitopenia en un solo caso, que cursó con un shock mixto, con miocarditis, insuficiencia cardiaca, meningitis aséptica y síndrome de activación macrofágica. La baja frecuencia de trombocitosis se explica porque la mayoría de las veces el recuento de plaquetas se realizó en la primera semana de evolución de la enfermedad. La elevación de reactantes de fase aguda (PCR y VHS) es universal en la EK, lo que concuerda con lo observado en nuestra serie. En este estudio hasta 1/3 de los pacientes registró alteración de las pruebas de función hepática, con elevaciones moderadas de transaminasas (GGT) e hiperbilirrubinemia. La hipoalbuminemia es común y se ha asociado a evoluciones más graves ${ }^{2}$. En nuestra serie, cuatro de los siete pacientes que presentaron EK grave presentaron hipoalbuminemia, lo que fue estadísticamente significativo. La piuria estéril se describe en $1 / 3$ de los pacientes; en nuestro análisis se observó sólo en 1/10 de los pacientes.

El compromiso cardíaco es la complicación más grave en la EK, con desarrollo de aneurismas coronarios que pueden causar infarto miocárdico y muerte súbita. Las lesiones coronarias generalmente no se presentan antes de los 10 días de evolución. El ecocardiograma debe realizarse siempre al momento del diagnóstico ya que, en la etapa aguda, el hallazgo de refringencia y/o dilatación de las arterias coronarias apoya la hipótesis diagnóstica de una EK. Otros hallazgos en esta etapa son disminución de la función ventricular, regurgitación valvular moderada y efusión pericárdica ${ }^{11}$. En casos no complicados, se recomienda repetir el ecocardiograma a las dos semanas y luego entre las seis y ocho semanas del inicio de la enfermedad ${ }^{2}$. En caso de fiebre persistente o hallazgos de alteraciones en la ecocardiografía de ingreso, se requiere un control más frecuente. El seguimiento ecocardiográfico debe identificar la progresión y regresión de anomalías coronarias, función valvular y ventricular y evolución del derrame pericárdico ${ }^{2}$.

A todos los pacientes de la serie presentada se les realizó al menos un ecocardiograma al ingreso; 37,5\% presentó alguna alteración ecocardiográfica, correspondiendo dos tercios a eco-refringencia o a dilatación coronaria. Durante la hospitalización hubo tres pacientes que desarrollaron aneurismas, uno durante la primera semana y dos durante la tercera. En suma, hubo compromiso de arterias coronarias en $34,3 \%$ de los casos, dilatación en $21,9 \%$ $\mathrm{y}$ aneurismas en $9,4 \%$. Estas cifras son concordantes con series coreanas y neozelandesas en la frecuencia de dilatación, pero no de aneurismas, siendo éstos más frecuentes en nuestra serie, lo que podría explicarse por el número menor de casos en nuestra serie respecto a las mencionadas ( 9.150 y 49 pacientes, respectivamente). En el presente trabajo no se analizó el seguimiento cardiológico de los pacientes. Por esto, y considerando que $81,3 \%$ de los niños estuvieron hospitalizados menos de una semana, no fue posible conocer el número total de aneurismas coronarios diagnosticados.

Entre 30 y 50\% de los pacientes con EK presentan vasculitis coronaria las primeras semanas de evolución, con moderada dilatación de estas arterias. Si el paciente es tratado sólo con ácido acetilsalicílico o si no recibe tratamiento, el riesgo de desarrollar aneurisma es de 20 a $25 \%$, versus 3 a $5 \%$ en pacientes que reciben inmunoglobulina ${ }^{3,9,12,13}$. Múltiples estudios demuestran que el tratamiento con IGIV (2 gr $/ \mathrm{kg}$ ) antes de 10 días desde el inicio de la fiebre, reduce significativamente la tasa de aneurismas coronarios ${ }^{2,5,14}$. El ácido acetilsalicílico en dosis altas no reduce la tasa de compromiso coronario; sin embargo, actúa sinérgicamente con la inmunoglobulina con efecto antiinflamatorio. La duración del tratamiento con ácido acetilsalicílico en altas dosis no está definido, pero se acepta la disminución a dosis de anti-agregante plaquetario (3-5 mg/kg/día) a las 48 horas si el paciente está afebril o luego de 14 días si el paciente presentó 
alteración en las arterias coronarias. Se mantendrá el fármaco por seis a ocho semanas luego del período de enfermedad activa (febril) o hasta la desaparición de las lesiones coronarias. La literatura científica describe que 8 a $23 \%$ de los pacientes requieren de una segunda dosis $^{5-12}$ de IGIV. En nuestra serie, la totalidad de los pacientes recibieron IGIV y $12,5 \%$ de los casos requirió una segunda dosis. De los que requirieron repetición de dosis de inmunoglobulina, $75 \%$ tenían 5 años de edad o más, hallazgo que se correlaciona con experiencias antes publicadas y que plantea aumento del riesgo de presentar co-morbilidad o mala evolución en pacientes de mayor edad ${ }^{15}$. Se han descrito nuevos tratamientos para casos refractarios, como corticosteroides, agentes citotóxicos (ciclofosfamida y ciclosporina), ulinastatina y anticuerpos monoclonales anti FNT- $\alpha$ (infliximab) ${ }^{16}$. Estudios han descrito que, en el total de pacientes con EK, la asociación de corticosteroides al tratamiento habitual no disminuiría la aparición de aneurismas coronarios; sin embargo, observaron que en aquellos pacientes con fiebre persistente, que requirieron de una segunda dosis de IGIV, la administración de metilprednisolona como tratamiento inicial, se correlacionó con menos lesiones coronarias ${ }^{16}$. Los agentes citotóxicos han sido utilizados con buenos resultados en series pequeñas de pacientes y el infliximab se mostró seguro y eficaz en un estudio retrospectivo de pacientes con EK refractaria al uso de IGIV y corticosteroides ${ }^{16}$. En nuestra serie, los casos considerados graves no requirieron estos tratamientos.

Es de interés comentar el caso de EK que se asoció a síndrome de activación macrofágica; hace ya algún tiempo que está descrita esta asociación en la literatura científica $^{17,18}$. Es una complicación poco frecuente, relacionada con una alteración de la regulación de los linfocitos $\mathrm{T}$, con excesiva producción de citoquinas y de hemofagocitos ${ }^{19-21}$. Se caracteriza por fiebre persistente, hepato-esplenomegalia, citopenias, disfunción hepática, coagulopatía, elevación de $\mathrm{LDH}$, hipertrigliceridemia, hiperferritinemia e hipofibrinogenemia ${ }^{19,20}$. El diagnóstico se basa en el examen citológico de la médula ósea, ganglio o hígado, el que muestra histiocitos benignos fagocitando en forma activa las células hematopoyéticas ${ }^{20}$. Habitualmente el cuadro es de rápida evolución y los pacientes son candidatos a recibir una segunda dosis de IGIV. En nuestro caso, el paciente presentó fiebre por nueve días, estuvo hospitalizado en la unidad de cuidados intensivos por siete días, cursando con un shock mixto, con coagulopatía, anemia, hipoalbuminemia y disfunción hepática, requiriendo de ventilación mecánica por cinco días y uso de fármacos vasoactivos. Pese a la gravedad del cuadro, no fue administrada una segunda dosis de IGIV.

Cabe destacar que, de los siete casos de EK grave, tres presentaron fiebre mayor a ocho días previo al diagnósti- co, uno desarrolló un aneurisma gigante con debut durante la primera semana y dos casos tuvieron hospitalización prolongada; en uno de ellos tardó en hacerse el diagnóstico 11 días desde su internación. Los factores de riesgo para EK graves encontrados en nuestra serie fueron edad mayor a 5 años y la presencia de hipoalbuminemia. Hasta hace algún tiempo, la tasa de letalidad de pacientes con EK era de 1 a $2 \%$. Actualmente, con el mayor conocimiento de la enfermedad, sospecha, diagnóstico y tratamiento precoz y más efectivo, se ha logrado reducir esta cifra a $0,04 \%$. En nuestra serie no se registraron muertes.

\section{Conclusión}

La EK es una enfermedad poco frecuente, pero de importancia en pediatría, dadas sus eventuales complicaciones. En la mayoría de los casos es de presentación típica, con predominio estacional en primavera y se concentra en niños bajo dos años de edad. Existen algunos parámetros que orientan a una evolución de mayor gravedad, como una edad mayor a 5 años y la hipoalbuminemia.

Agradecimientos. A Lorena Porte, Marcela Paredes y Marcos Emilfork.

\section{Resumen}

Introducción: La enfermedad de Kawasaki (EK) es una afección grave en pediatría, por sus eventuales complicaciones y secuelas si no es tratada adecuada y precozmente. Objetivos: Describir el perfil clínico-epidemiológico de los niños hospitalizados en una clínica de la Región Metropolitana e identificar factores de riesgo para evolución desfavorable. Pacientes y Métodos: Estudio descriptivo, retrospectivo, de 32 pacientes hospitalizados en la Clínica Santa María con diagnóstico de egreso de EK entre febrero de 1999 y mayo de 2007. Resultados: La frecuencia anual fue de 5 casos, con predominio en primavera y en el sexo masculino. La mediana para la edad fue de 1,5 años, $87,5 \%$ tenían menos de 5 años. Predominó la presentación típica $(68,7 \%)$. Hubo dilatación y/o aneurisma de arterias coronarias en 21,9\%, con 3 casos de aneurismas. El tratamiento se realizó con inmunoglobulina intravenosa (IGIV) y ácido acetilsalicílico. Cuatro pacientes requirieron una segunda dosis de IGIV. No hubo fallecidos. Como factores de riesgo para mala evolución se encontraron la edad de presentación sobre 5 años y la hipoalbuminemia. Conclusiones: La EK es una patología poco frecuente, predominante en niños bajo 5 años de edad y con presentación típica. Sugieren evolución de mayor gravedad la presentación sobre los 5 años y la hipoalbuminemia a cualquier edad. 


\section{Referencias}

1.- Rowley A, Schulman S. Capítulo 166. Enfermedad de Kawasaki, Nelson. Tratado de Pediatría, $16^{\circ}$ ed. México. McGraw-Hill Interamericana 2001; p. 795-7.

2.- Newburger J, Takahashi M, Gerber M, Gewitz M, Tani L, Burns J, et al. Diagnosis, treatment, and long-term management of Kawasaki disease: a statement for health professionals from the Committee of Rheumatic Fever, Endocarditis, and Kawasaki Disease, Council on Cardiovascular Disease in the Young, American Heart Association. Pediatrics 2004; 114: 1708- 33.

3.- Freeman A, Schulman S. Kawasaki disease: Summary of the American Heart Association Guidelines 2006; 74: n.7.

4.- Yanagawa H, Nakamura Y, Yashir M, Oki I, Hirata S, Zhang T, et al. Incident survey of Kawasaki disease in 1997 and 1998 in Japan. Pediatrics 2001; v.107 n.3.

5.- Grewal D, Wong M, Isaacs D. Diagnosis, treatment and outcome of Kawasaki disease in an Australian tertiary setting: A review of three year experience. J Paediatr Child Health 2005; 41: 495-99.

6.- Cordero J, Banfi A, Wu E, Enríquez G. Epidemiologic and clinical characteristics of Kawasaki disease in Chile. In: Kawasaki
Disease. Editor H. Kato. Elsevier Science B.V. 1995: 53-61.

7.- Schonhaut L, Herrera P, Acevedo K, Álvarez P. Enfermedad de Kawasaki en Hospital Roberto del Río: análisis clínico epidemiológico. Rev Chil Pediatr 2001; v.72 n.4.

8.- Oates-Whitehead R, Baumer J, Haines L, Love S, Maconochie IK, Gupta A, et al. Intravenous immunoglobulin for the treatment of Kawasaki disease in children. Cochrane Database of Systematic Reviews. 2003;4:CD004000.

9.- Won J, Whan H, Chang K, Soo Y, Ho S, Sook J, et al. Epidemiologic picture of Kawasaki disease in Korea, 2000-2002. Pediatrics Internat 2005; 47: 382-7.

10.- Heaton P, Wilson N, Nicholson R, Doran J, Parsons A, Aiken J. Kawasaki disease in New Zeland. J Pediatr Child Health 2006; v. $42: 184-90$

11.- Banfi A, Olguín H, Herrera P, Enríquez G, Talesnik E. Enfermedad de Kawasaki. Rev Chil Pediatr 1992; 63: 347-52.

12.- Burns J: The riddle of Kawasaki disease. N Engl Med J 2007; 356 (7): 659-61.

13.- Rowley A, Schulman S: Kawasaki syndrome. Clin Microbiol Rev 1998; 11 (3): 405-14.

14.- Tamura T, Tomomasa T, Kobayashi T, Morikawa A, Kobayashi T, Inoue Y, et al. Prediction of intravenous immunoglobulin unresponsiveness in patients with Kawasaki disease. Circulation 2006; 113: 2602-12.

15.- Momenah T, Sanatani S, Potts J, Sandor G G, Human D G, Patterson M W. Kawasaki disease in the older child. Pediatrics 1998: 102-7.

16.- Prieto L, Cuesta M, Guillén S, Ruiz M, Cortés M, Rubio B, et al. Enfermedad de Kawasaki: diagnóstico y tratamiento. An Pediatr (Barc) 2010; 73 (1): 25-30.

17.- Ohga S, Ooshima A, Fukushige, Ueda K. Histocytic haemophagocytosis in a patient with Kawasaki disease: changes in the hypercytokinaemic state. Eur J Pediatr 1995; 154: 539-41.

18.- Kaneko K, Takahashi K, Fujiwara S, Maluynma T, Obinata K. Kawasaki disease followed by hemophagocytic syndrome. Acta Pediatr 1998; 610-11.

19.- Muise A, Tallett S, Silverman E. Are children with Kawasaki disease and prolonged fever at risk for macrophage activation syndrome? Pediatrics 2003; 112; e495-7.

20.- Wea'am Al-Ei, Abdullah Al-Jefri, Sultan Bahabri. Hemophagocytosis complicating Kawasaki disease. Pediatr Hematol Oncol 2000; 17: 323-9.

21.- Verdugo P, Rodríguez N, Tordecilla J, Soto V. Síndrome hemofagocítico secundario en pediatría: Experiencia clínica en ocho casos. Rev Chil Pediatr 2005; 76 (4); 397-403. 